\title{
On the Motion of the Chaplygin Sleigh on a Horizontal Plane with Dry Friction at Three Points of Contact
}

\begin{abstract}
A. Yu. Shamin
This paper addresses the problem of the motion of the Chaplygin sleigh, a rigid body with three legs in contact with a horizontal plane, one of which is equipped with a semicircular skate orthogonal to the horizontal plane. The problem is considered in a nonholonomic setting: assuming that the blade cannot slide in a direction perpendicular to its plane, but unlike the Chaplygin problem, there is a dry friction force in the skate that is directed along the skate, along which the blade plane and the reference plane intersect. It is also assumed that at the two other points of support there are dry friction forces.

The equations of motion of the Chaplygin sleigh are obtained, and a number of properties are proved. It is proved that the movement ceases in finite time. The possibility of realizing the nonnegativity of normal reactions is discussed. The case of static friction is studied when the blade velocity is $v=0$. A region of stagnation where the system rotates about a fixed vertical axis is found. On this set, the equations of motion are integrated and the law of variation of the angular velocity is found. Examples of trajectories of the sleigh are given. A qualitative description of the motion is obtained: the behavior of the phase curves in a neighborhood of the equilibrium point is investigated depending on the geometric and mass characteristics of the system.
\end{abstract}

Keywords: dry friction, Chaplygin sleigh

Received February 25, 2019

Accepted April 23, 2019

This work was supported by the Russian Foundation for Basic Research (19-01-00140).

Alexander Yu. Shamin

shamin_ay@mail.ru

Moscow State University,

Vorobievy gory 1, Moscow, 119899 Russia

RUSSIAN JOURNAL OF NONLINEAR DYNAMICS, 2019, 15(2), 159-169 


\section{Introduction}

This paper is concerned with the problem of the motion of the Chaplygin sleigh, a rigid body with three legs in contact with a horizontal plane, one of which is equipped with a semicircular knife edge orthogonal to the supporting plane. The problem is considered in a nonholonomic setting, under the assumption that the knife edge cannot slide in the direction perpendicular to its plane, but, in contrast to the Chaplygin problem, the skate has dry friction force, which is directed along the skate, where the plane of the knife edge and the supporting plane intersect. It is also assumed that the body is acted upon by dry friction forces at two other supporting points as well.

In the absence of friction at the points of contact of the body with the plane the problem under consideration becomes the Chaplygin problem [1,2], and in the absence of a knife edge and in the presence of friction at all points of contact it becomes the problem of the motion of a tripod on a plane with friction [3]. The case where there is no friction at the point of contact of the knife edge with the plane is dealt with in [4]. At present, problems concerning the mechanics of systems with friction are becoming topics of much current interest. Mention should also be made of Refs. [5-7], which investigate various mechanical systems with dry friction.

In this paper, equations of motion of the system of interest are written out, a qualitative analysis of the body motion is given, and the phase portrait of the system in a neighborhood of an equilibrium point is investigated.

\section{Formulation of the problem}

Consider a rigid body moving with three points $A, B$ and $C$ in contact with a horizontal plane (see Fig. 1). The body is an isosceles triangle $(A C=B C, C$ being the supporting point of the knife edge). It is assumed that the plane of the knife edge is orthogonal to the supporting plane and intersects with the latter along the symmetry axis $E C$ of the triangle $A B C$, and the center of mass $S$ of the body is projected into the point $D$, which lies on this axis $(A E=E B=a, E D=b, D C=c, D S=h)$.

Let $O x y z$ be a fixed coordinate system $(O x y$ being the supporting plane and $O z$ the upward vertical) and let $S \xi \eta \zeta$ be the principal central axes of inertia of the body, with $S \zeta \| O z$. Let $\vec{e}_{x}, \vec{e}_{y}, \vec{e}_{z}$ and $\vec{e}_{\xi}, \vec{e}_{\eta}, \vec{e}_{\zeta}$ denote the unit vectors of the axes $O x y z$ and $S \xi \eta \zeta$, respectively:

$$
\vec{e}_{\xi}=\vec{e}_{x} \cos \psi+\vec{e}_{y} \sin \psi, \vec{e}_{\eta}=-\vec{e}_{x} \sin \psi+\vec{e}_{y} \cos \psi, \vec{e}_{\zeta}=\vec{e}_{z}
$$

where $\psi$ is the angle between the axes $O x$ and $S \xi$.

Assume that the velocity of the point $C$ of the body is $\vec{v}_{C}=v \vec{e}_{\xi}$ (the body cannot slide in the direction orthogonal to the plane of the knife edge), and the angular velocity of the body is $\vec{\omega}=\omega \vec{e}_{\zeta}(\omega=\dot{\psi})$, that is, the body can rotate only about the vertical passing through point $C$.

If the body moves without loss of contact with the plane, the velocity $\vec{v}_{S}$ of its center of mass and the velocities $\vec{v}_{A}, \vec{v}_{B}$ of its supporting points $A, B$ are defined by

$$
\begin{gathered}
\vec{v}_{S}=\vec{v}_{C}+[\vec{\omega}, \overrightarrow{C S}]=v \vec{e}_{\xi}-\omega c \vec{e}_{\eta}, \\
\vec{v}_{A}=\vec{v}_{C}+[\vec{\omega}, \overrightarrow{C A}]=(v+a \omega) \vec{e}_{\xi}-(b+c) \omega \vec{e}_{\eta}, \\
\vec{v}_{B}=\vec{v}_{C}+[\vec{\omega}, \overrightarrow{C B}]=(v-a \omega) \vec{e}_{\xi}-(b+c) \omega \vec{e}_{\eta} .
\end{gathered}
$$




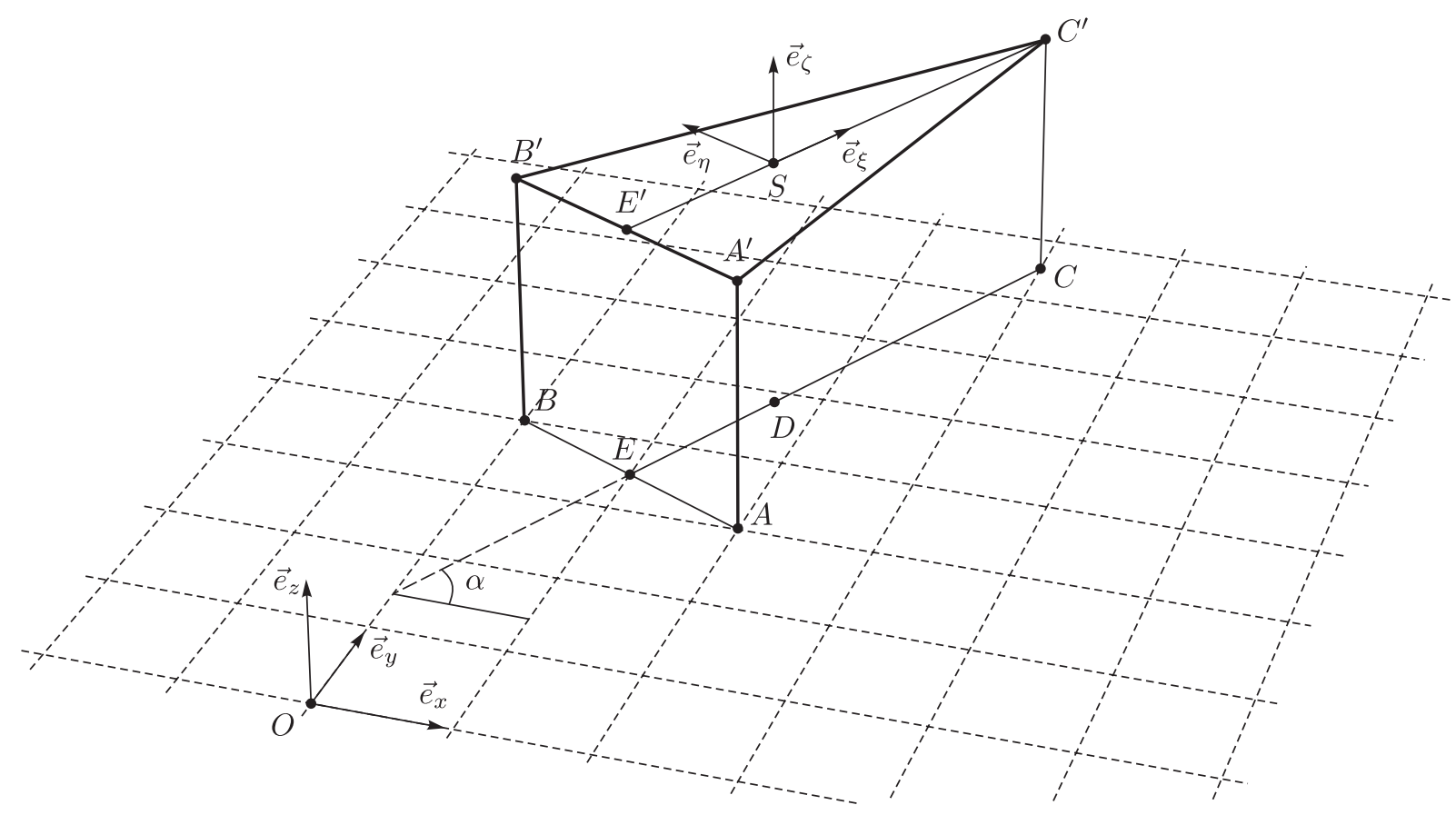

Fig. 1. The Chaplygin sleigh.

The body is acted upon by the gravity force $\vec{P}=-m g \vec{e}_{\zeta}$ ( $m$ is the mass of the body and $g$ is the free-fall acceleration), which is applied at point $S$, and by the reaction forces $\vec{R}_{A}, \vec{R}_{B}, \vec{R}_{C}$ applied at points $A, B, C$ :

$$
\begin{gathered}
\vec{R}_{A}=N_{A} \vec{e}_{\zeta}-k N_{A} \frac{\vec{v}_{A}}{v_{A}}, \vec{R}_{B}=N_{B} \vec{e}_{\zeta}-k N_{B} \frac{\vec{v}_{B}}{v_{B}}, \vec{R}_{C}=N_{C} \vec{e}_{\zeta}+F_{\text {fr }}(v) \vec{e}_{\xi}+R \vec{e}_{\eta}, \\
F_{\text {fr }}(v)=\left\{\begin{array}{l}
-k_{C} N_{C} \frac{v}{v_{C}}, v \neq 0, \\
\theta \in\left[-k_{C} N_{C} ; k_{C} N_{C}\right], v=0 .
\end{array}\right.
\end{gathered}
$$

Here $N_{A}, N_{B}, N_{C}$ are the normal reactions at the points of support, $k>0$ is the coefficient of Coulomb friction at the points $A, B$ of contact of the legs of the sleigh with the plane, $k_{C}>0$ is the coefficient of Coulomb friction for the knife edge at point $C$ (in the general case $\left.k \neq k_{C}\right)$, and $R$ is the reaction of the nonholonomic constraint $\left(\vec{v}_{C}, \vec{e}_{\eta}\right)=0, v_{A}=$ $=\sqrt{(v+a \omega)^{2}+(b+c)^{2} \omega^{2}}, v_{B}=\sqrt{(v-a \omega)^{2}+(b+c)^{2} \omega^{2}}, v_{C}=|v|$.

\subsection{Definition of reactions}

The principal vector of forces that acts on the body has the form $\vec{F}=F_{\xi} \vec{e}_{\xi}+F_{\eta} \vec{e}_{\eta}+F_{\zeta} \vec{e}_{\zeta}$,

$$
\begin{gathered}
F_{\xi}=-k\left(\frac{N_{A}}{v_{A}}+\frac{N_{B}}{v_{B}}\right) v+F_{\mathrm{fr}}(v)+k\left(\frac{N_{B}}{v_{B}}-\frac{N_{A}}{v_{A}}\right) a \omega, \\
F_{\eta}=k\left(\frac{N_{A}}{v_{A}}+\frac{N_{B}}{v_{B}}\right)(b+c) \omega+R, \\
F_{\zeta}=N_{A}+N_{B}+N_{C}-m g .
\end{gathered}
$$


Let us find the principal vector of the torques acting on the body relative to its center of mass

$$
\begin{gathered}
\vec{M}=\left[\overrightarrow{S A}, \vec{R}_{A}\right]+\left[\overrightarrow{S B}, \vec{R}_{B}\right]+\left[\overrightarrow{S C}, \vec{R}_{C}\right]=M_{\xi} \vec{e}_{\xi}+M_{\eta} \vec{e}_{\eta}+M_{\zeta} \vec{e}_{\zeta}, \\
M_{\xi}=a\left(N_{B}-N_{A}\right)+k h\left(\frac{N_{A}}{v_{A}}+\frac{N_{B}}{v_{B}}\right)(b+c) \omega+h R, \\
M_{\eta}=b\left(N_{A}+N_{B}\right)-c N_{C}+k h\left(\frac{N_{A}}{v_{A}}+\frac{N_{B}}{v_{B}}\right) v-F_{\text {fr }}(v) h-k h\left(\frac{N_{B}}{v_{B}}-\frac{N_{A}}{v_{A}}\right) a \omega, \\
M_{\zeta}=k\left(\frac{N_{B}}{v_{B}}-\frac{N_{A}}{v_{A}}\right) a v-k\left(\frac{N_{A}}{v_{A}}+\frac{N_{B}}{v_{B}}\right)\left(l^{2}-c(b+c)\right) \omega+c R,
\end{gathered}
$$

where $l^{2}=a^{2}+(b+c)^{2}$. Thus, projected onto the principal central axes of inertia of the body, the equations of motion of the body in the Newton-Euler form which take into account the conditions of motion without loss of contact of the body with the plane $\left(\left(\vec{v}_{S}, \vec{e}_{\xi}\right)=0,\left(\vec{\omega}, \vec{e}_{\xi}\right)=\right.$ $=\left(\vec{\omega}, \vec{e}_{\eta}\right)=0$ ) have the form ( $J$ is the moment of inertia of the body relative to the axis $S \zeta)$

$$
\begin{gathered}
m\left(\dot{v}+c \omega^{2}\right)=F_{\xi}, \quad m(v \omega-c \dot{\omega})=F_{\eta}, \quad 0=F_{\zeta}, \\
0=M_{\xi}, \quad 0=M_{\eta}, J \dot{\omega}=M_{\zeta} .
\end{gathered}
$$

The system (2.1)-(2.2) is closed relative to the variables $v, \omega, N_{A}, N_{B}, N_{C}$ and $R$. The reactions $N_{C}$ and $R$ can be expressed in terms of the reactions $N_{A}$ and $N_{B}\left(N_{C}\right.$ can be expressed from the third equation of (2.1) and $R$ from the second equation of (2.1) with the third equation of (2.2) taken into account):

$$
\begin{gathered}
N_{C}=m g-\left(N_{A}+N_{B}\right), \\
R=\left[J m v \omega+k\left(m c l^{2}-I(b+c)\right) \omega\left(\frac{N_{A}}{v_{A}}+\frac{N_{B}}{v_{B}}\right)-k m a c v\left(\frac{N_{B}}{v_{B}}-\frac{N_{A}}{v_{A}}\right)\right] I^{-1}, \\
I=J+m c^{2} .
\end{gathered}
$$

The reactions $N_{A}$ and $N_{B}$ are uniquely defined from the system

$$
\begin{aligned}
& (b+c)\left(N_{A}+N_{B}\right)+k h v\left(\frac{N_{A}}{v_{A}}+\frac{N_{B}}{v_{B}}\right)-F_{\mathrm{fr}}(v) h-k h a \omega\left(\frac{N_{B}}{v_{B}}-\frac{N_{A}}{v_{A}}\right)=m g c, \\
& -I a\left(N_{B}-N_{A}\right)+m a c k h v\left(\frac{N_{B}}{v_{B}}-\frac{N_{A}}{v_{A}}\right)-m l^{2} c k h \omega\left(\frac{N_{A}}{v_{A}}+\frac{N_{B}}{v_{B}}\right)=J m v \omega h,
\end{aligned}
$$

which is obtained from the first two equations of (2.2) with (2.4) taken into account. Obviously, the reactions $N_{A}, N_{B}$ (and hence $N_{C}, R$ ) depend only on the phase variables $v, w$ and the system parameters.

We note that the solution of the system (2.5)-(2.6) must satisfy the conditions

$$
N_{A} \geqslant 0, \quad N_{B} \geqslant 0, \quad N_{A}+N_{B} \leqslant m g,
$$

which ensure (along with conditions (2.3), (2.5), and (2.6)) that the body moves without loss of contact with the plane. Conditions (2.7) impose a restriction on the height of the center of mass of the body. Indeed, if we fix the mass and geometric parameters of the sleigh, except for $h$, 
with $b, c>0$, and take arbitrary $\omega_{0}, v_{0}$, then from Eqs. (2.5), (2.6) we obtain normal reactions $N_{A}(h), N_{B}(h)$ as functions of height. It is obvious that

$$
N_{A}(0)=N_{B}(0)=\frac{m g c}{2(b+c)}
$$

and condition (2.7) is satisfied, with all inequalities being rigorous. But since $N_{A}(h), N_{B}(h)$ are continuous at $h=0$, conditions (2.7) will be satisfied also when $\forall h \in\left[0 ; h_{0}\right)$ for some $h_{0}$ depending on the initial velocities $\omega_{0}, v_{0}$. An example of normal reactions at the points of contact is given in Fig. 2.

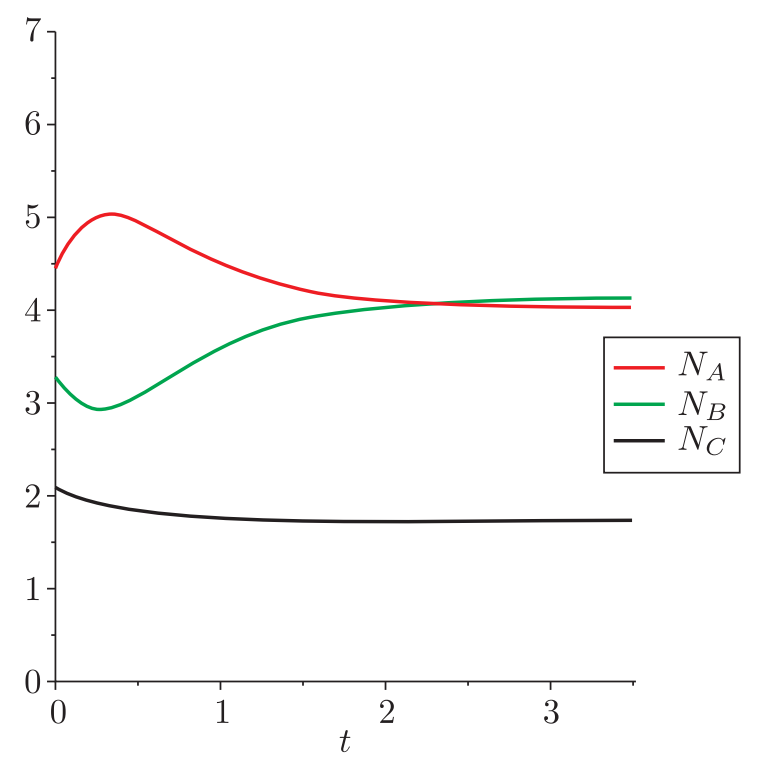

Fig. 2. Reactions $N_{A}(t), N_{B}(t), N_{C}(t)$.

The set $M=\left\{(v, \omega): T(v, \omega) \leqslant T\left(v_{0}, \omega_{0}\right)\right\}$ is compact. Then such a value $h_{0}$ can be chosen simultaneously for all $(v, \omega) \in M$. Thus, there exists $h_{0}>0$, which depends only the system parameters and the initial velocities $v_{0}, \omega_{0}$, such that, when $h<h_{0}$, conditions (2.7) are satisfied for all values of $v, \omega$ at which the corresponding kinetic energy does not exceed the initial one.

\section{Equations of motion and properties of their solutions}

Let $h<h_{0}$. Then the motion of the Chaplygin sleigh on the horizontal plane with dry friction at the supporting points $A$ and $B$ is described by a second-order system relative to the phase variables of the problem, $v$ and $\omega$.

$$
\begin{gathered}
m \dot{v}+m c \omega^{2}=-k\left(\frac{N_{A}}{v_{A}}+\frac{N_{B}}{v_{B}}\right) v+F_{\mathrm{fr}}(v)+k\left(\frac{N_{B}}{v_{B}}-\frac{N_{A}}{v_{A}}\right) a \omega, \\
I \dot{\omega}-m c v \omega=-k\left(\frac{N_{A}}{v_{A}}+\frac{N_{B}}{v_{B}}\right) l^{2} \omega+k\left(\frac{N_{B}}{v_{B}}-\frac{N_{A}}{v_{A}}\right) a v .
\end{gathered}
$$

These equations are obtained from the first equation of (2.1) and from the third equation of (2.2) with (2.3) and (2.4) taken into account, and $N_{A}(v, \omega)$ and $N_{B}(v, \omega)$ are defined from the system $(2.5)-(2.6)$. 


\subsection{The case $v=0$}

We first consider the case of rest of the point of contact, $C$, of the knife edge. In this case, we set $v=0, \dot{v}=0$. Then Eqs. (2.5), (2.6), (3.1), and (3.2) can be rewritten as

$$
\begin{gathered}
(b+c)\left(N_{A}+N_{B}\right)-\theta h-\frac{k h a}{l} \frac{\omega}{|\omega|}\left(N_{B}-N_{A}\right)=m g c, \\
-I a\left(N_{B}-N_{A}\right)-m l c k h \frac{\omega}{|\omega|}\left(N_{A}+N_{B}\right)=0, \\
m c \omega^{2}=\theta+\frac{k a}{l}\left(N_{B}-N_{A}\right) \frac{\omega}{|\omega|}, \\
I \dot{\omega}=-k l\left(N_{A}+N_{B}\right) \frac{\omega}{|\omega|},
\end{gathered}
$$

where $|\theta| \leqslant k_{C} N_{C}$. From the first three equations, with (2.3) taken into account, one can find conditions under which point $C$ will remain at rest, as well as the values of $\theta, N_{A}$, and $N_{B}$.

Statement 1. The system (3.1), (3.2) admits an invariant set $M=\{(\omega, v): v=0$, $\omega^{2} \leqslant \frac{g\left(I b k_{C}-m c^{2} k^{2} h\right)}{c I(b+c)+m c^{2} k^{2} h^{2}+k_{C} c h I}$.

Proof.

If $v=0$ and $\dot{v}=0$, then $v_{A}=v_{B}=l|\omega|$, hence $N_{A}=N_{0}(1+\nu), N_{B}=N_{0}(1-\nu)$, where

$$
N_{0}=\frac{m c\left(g+\omega^{2} h\right)}{2(b+c)}, \quad \nu=\frac{m l c k h}{I a} \frac{\omega}{|\omega|} .
$$

In this case,

$$
\theta=m c \omega^{2}+\frac{m^{2} c^{2} k^{2} h\left(g+\omega^{2} h\right)}{I(b+c)} .
$$

In view of (2.3) the condition $|\theta| \leqslant k_{C} N_{C} \Leftrightarrow$ is

$$
\omega^{2} \leqslant \frac{g\left(I b k_{C}-m c^{2} k^{2} h\right)}{c I(b+c)+m c^{2} k^{2} h^{2}+k_{C} c h I} .
$$

It should be noted that the set exists under the condition $k_{C}>\frac{m c^{2} h k}{I b}$. Consider the motion on the invariant set $M$. Given the previous statement, the equation $I \dot{\omega}=-k l\left(N_{A}+N_{B}\right) \frac{\omega}{\omega}$ has the form

$$
\dot{\omega}=-\frac{m l c k\left(g+\omega^{2} h\right)}{I(b+c)} \frac{\omega}{|\omega|} .
$$

Integrating it for $\omega>0$, we obtain

$$
\omega=\sqrt{\frac{g}{h}} \tan \left(\arctan \left(\sqrt{\frac{h}{g}} \omega_{0}\right)-\frac{m c l k \sqrt{h g}}{I(b+c)} t\right) .
$$




\subsection{The case $v \neq 0$}

Consider the instants of time when the velocity of the point of the skate, $C$, does not vanish.

Statement 2. The system (3.1)-(3.2) is invariant under the change of $\omega$ to $-\omega$.

Proof.

In the case of this replacement, $v_{A}$ and $v_{B}$ are interchanged. Consequently (see $(2.5)$ and (2.6)), $N_{A}$ and $N_{B}$ are interchanged, i.e., the system (3.1)-(3.2) maps into itself.

Statement 3. The system (3.1)-(3.2) admits an invariant set $\omega \equiv 0$ along which the motion of the Chaplygin sleigh is defined by the following relations for $v_{0}>0$ and $v_{0}<0$, respectively:

$$
\begin{aligned}
& v(t)=v_{0}-\frac{g\left(c k+b k_{C}\right)}{(b+c)+h\left(k-k_{C}\right)} t, \quad t \in\left[0, t_{+}\right] ; t_{+}=\frac{\left((b+c)+h\left(k-k_{C}\right)\right) v_{0}}{g\left(c k+b k_{C}\right)} \\
& v(t)=v_{0}+\frac{g\left(c k+b k_{C}\right)}{(b+c)-h\left(k-k_{C}\right)} t, \quad t \in\left[0, t_{-}\right] ; t_{-}=\frac{\left((b+c)-h\left(k-k_{C}\right)\right)\left|v_{0}\right|}{g\left(c k+b k_{C}\right)}
\end{aligned}
$$

$\left(v_{0}=v(0)\right.$; without loss of generality the initial instant of time is assumed to be zero).

Proof.

When $\omega=0$, Eq. (3.2) is satisfied identically for any $v$ and Eq. (3.1) takes the form (see $(2.8))$

$$
\dot{v}=-\frac{g\left(c k+b k_{C}\right)}{b+c+\left(k-k_{C}\right) h \frac{v}{|v|}} \frac{v}{|v|} .
$$

If $v_{0}>0$, then $\dot{v}=-g\left(c k+b k_{C}\right)\left(b+c+h\left(k-k_{C}\right)\right)^{-1}$, and if $v_{0}<0$, then $\dot{v}=g\left(c k+b k_{C}\right)(b+$ $\left.+c-h\left(k-k_{C}\right)\right)^{-1}$, from which relations (3.4) and (3.5) follow immediately.

Remark 1. Obviously, $t_{+}>t_{-} \Leftrightarrow k>k_{C}$, i..e., the translational motion of the Chaplygin sleigh during which the knife edge is ahead lasts longer than in the case where the knife edge is behind if and only if the coefficient of dry friction in the skate is less than the coefficients of friction in the legs (the absolute value of the initial velocity being the same).

Statement 4. The motion of the Chaplygin sleigh on a horizontal plane with dry friction at any initial values $v_{0}$ and $\omega_{0}$ which are not zero simultaneously ceases in finite time (if $v_{0}=0$, $\omega_{0}=0$, then $\left.v \equiv 0, \omega \equiv 0\right)$.

Proof.

It follows from the system (3.1)-(3.2) that

$$
\dot{T}=-k Q(v, \omega)
$$

where $T=\frac{m v^{2}+I \omega^{2}}{2}$ is the kinetic energy,

$$
Q=\left(\frac{N_{A}}{v_{A}}+\frac{N_{B}}{v_{B}}+\frac{k_{C} N_{C}}{k v_{C}}\right) v^{2}-2\left(\frac{N_{B}}{v_{B}}-\frac{N_{A}}{v_{A}}\right) v a \omega+\left(\frac{N_{A}}{v_{A}}+\frac{N_{B}}{v_{B}}\right) l^{2} \omega^{2} .
$$

Obviously, the $Q$-homogeneous function is a function of degree 1 of phase variables $v$ and $\omega$, and $Q>0$ for any $v$ and $\omega$ that are not zero simultaneously. Consequently, there exists a constant $q>0$ such that $Q \geqslant q \sqrt{T}$. In this case, relation (3.6) takes the form

$$
\dot{T}=\frac{d(\sqrt{T})^{2}}{d t}=2 \sqrt{T} \frac{d \sqrt{T}}{d t}=-k Q \leqslant-k q \sqrt{T}
$$


and hence $\frac{d \sqrt{T}}{d t} \leqslant-\frac{k q}{2}$, i.e., $\sqrt{T} \leqslant \sqrt{T_{0}}-\frac{k q t}{2} \quad\left(T_{0}=\frac{m v_{0}^{2}+I \omega_{0}^{2}}{2}\right)$. Taking into account the fact that $\sqrt{T} \geqslant 0$, we conclude that the motion ceases in finite time $t_{0} \leqslant \frac{2 \sqrt{T_{0}}}{k q}$.

\section{The phase portrait for $h=0$}

If $h=0$ and $v \neq 0$, Eqs. (3.1) and (3.2) take the form

$$
\begin{gathered}
\dot{v}=-c \omega^{2}-\frac{k g c}{2(b+c) v_{A} v_{B}} V-k_{C} N_{C} \frac{v}{|v|}, V=\left(v_{A}+v_{B}\right) v-\left(v_{A}-v_{B}\right) a \omega, \\
I \dot{\omega}=m c v \omega+\frac{m k g c}{2(b+c) v_{A} v_{B}} \Omega, \Omega=\left(v_{A}-v_{B}\right) a v-\left(v_{A}+v_{B}\right) l^{2} \omega .
\end{gathered}
$$

Obviously, the system (4.1)-(4.2) admits the solution (cf. (3.4), (3.5))

$$
\omega \equiv 0, \quad v=v_{0}-\operatorname{sign}\left(v_{0}\right) \frac{g\left(k c+b k_{C}\right)}{b+c} t \quad\left(t \in\left[0, t_{0}\right], \quad t_{0}=\frac{(b+c)\left|v_{0}\right|}{g\left(k c+b k_{C}\right)}\right)
$$

and, in particular, the solution $\omega \equiv 0, v \equiv 0$. An example of a phase portrait of the system is given in Fig. 5a.

The system admits motions along the phase trajectory $\omega=0$. In this case, the trajectory of the skate and of the center of mass is a segment, and the system executes translational motion. If the value of the initial velocity of the skate $v$ is nonzero, the motion of the sleigh can cease in the stagnation region (3.3). 2 cases can arise. If the initial value of $|\omega|$ is relatively small, velocity $v$ decreases monotonically to zero and gets into the stagnation zone, and the system begins to rotate about a fixed vertical axis passing through the skate (Fig. 3a). The other case of motion is possible when the absolute value of the initial angular velocity is sufficiently large. Then, monotonically decreasing, velocity $v$ reaches zero, but the phase curve does not get into the stagnation region, and the system starts moving back, after which the absolute value of velocity $v$ increases for some time and then begins to decrease and the system arrives at the stagnation region (an example of the trajectory is given in Fig. 3b). In this case, the trajectory of the skate is a "beak" at the point where $v=0$.

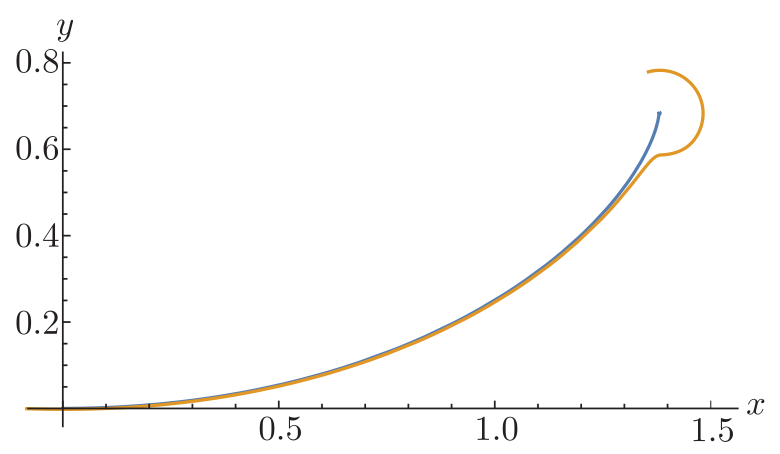

(a)

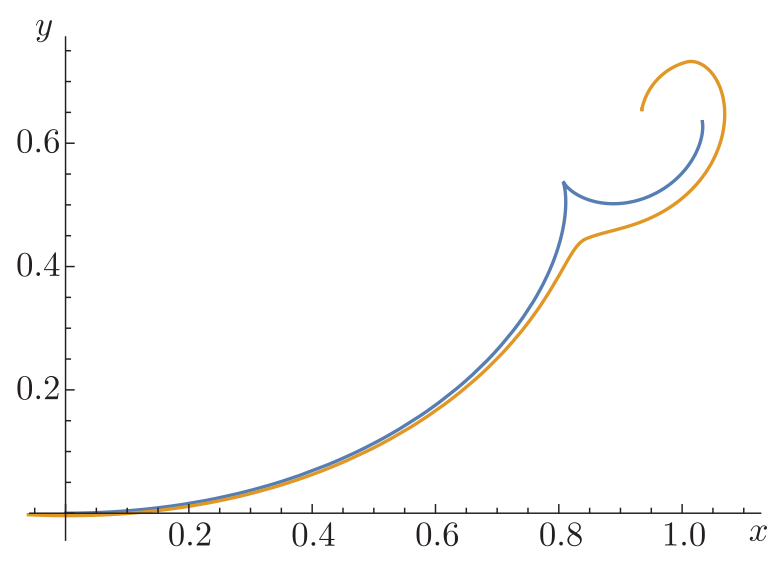

(b)

Fig. 3. Examples of trajectories. 
Let us investigate the behavior of the phase curves in a neighborhood of an equilibrium point. We consider the case $\omega>0$ and introduce a small parameter $\varepsilon$ and new variables:

$$
\varepsilon^{2}=\frac{v_{0}^{2}+\left(a \omega_{0}\right)^{2}}{a g}, v=\varepsilon \widetilde{v}, \omega=\varepsilon \widetilde{\omega}, t=\varepsilon \widetilde{t}, z=\frac{v}{a \omega}=\frac{\widetilde{v}}{a \widetilde{\omega}}
$$

Introducing the new notation $\mu^{2}=\frac{I}{m a^{2}}, \lambda=\frac{b+c}{a}$, we rewrite Eqs. (4.1), (4.2) as

$$
\begin{aligned}
& \frac{d \widetilde{v}}{d t}=-\varepsilon c \widetilde{\omega}^{2}-\frac{k g c}{2(b+c)} \frac{A(z) z+B(z)}{W(z)}-\frac{k_{C} g b}{b+c} \operatorname{sign}(z), \\
& \frac{d(a \widetilde{\omega})}{d t}=c \varepsilon^{2} \widetilde{\omega}^{2} \frac{z}{\mu^{2}}-\frac{k g c}{2(b+c) \mu^{2}} \frac{\left(1+\lambda^{2}\right) A(z)+B(z) z}{W(z)},
\end{aligned}
$$

where

$$
\begin{gathered}
\varkappa(z)=\frac{k_{C} b}{k c} \operatorname{sign}(z), \quad w_{A, B}(z)=\sqrt{(1 \pm z)^{2}+\lambda^{2}} \\
A(z)=w_{A}(z)+w_{B}(z), B(z)=w_{B}(z)-w_{A}(z), W(z)=w_{A}(z) w_{B}(z) .
\end{gathered}
$$

Rescaling time as $\tau=\ln \frac{\widetilde{\omega}_{0}}{\widetilde{\omega}}, \tau \in[0 ;+\infty)$, we obtain

$$
\begin{aligned}
\frac{d z}{d \tau}= & \frac{B(z) z^{2}+\left(1+\lambda^{2}-\mu^{2}\right) A(z) z-\mu^{2}(B(z)+2 \varkappa W(z))}{\left(1+\lambda^{2}\right) A(z)+B(z) z}- \\
& -\varepsilon^{2} \frac{2 \mu^{2}(b+c) W(z)}{k g} \widetilde{\omega}_{0}^{2} e^{-2 \tau} \frac{\left(1+\lambda^{2}\right) A(z)+2(B(z) z+\varkappa W(z))+A(z) z^{2}}{\left.\left[(1+\lambda)^{2} A(z)+B(z) z\right)\right]^{2}} .
\end{aligned}
$$

Consider the first approximation of Eq. (4.8) in the small parameter $\varepsilon$ :

$$
\frac{d z}{d \tau}=\frac{B(z) z^{2}+\left(1+\lambda^{2}-\mu^{2}\right) A(z) z-\mu^{2}(B(z)+2 \varkappa W(z))}{\left(1+\lambda^{2}\right) A(z)+B(z) z}=C(z) .
$$

Obviously, the right-hand side of (4.8) is an odd function, and so, in what follows, we will consider only the case $z>0$. It is also easy to verify that $\left(1+\lambda^{2}\right) A(z)+B(z) z>0 \forall z$. have

Let us check whether $u=\frac{a \widetilde{\omega}}{\tilde{v}}=\frac{1}{z}=0$ is a solution. Then, taking $u \geqslant 0$ into account, we

$$
\begin{gathered}
\frac{d u}{d \tau}=-\frac{1}{z^{2}} C\left(\frac{1}{u}\right)=D(u), \\
D(u)=-\frac{E(u)+\left(1+\lambda^{2}-\mu^{2}\right) F(u) u-\mu^{2}\left(E(u) u^{2}+u \varkappa \frac{F^{2}(u)-E^{2}(u)}{2}\right)}{\left(1+\lambda^{2}\right) F(u)+\frac{E(u)}{u}} .
\end{gathered}
$$

Here

$$
E(u)=B\left(\frac{1}{u}\right) u, F(u)=A\left(\frac{1}{u}\right) u
$$

since

$$
E(0)=0, F(0)=2, \lim _{u \rightarrow 0} \frac{E(u)}{u}=-2 \Rightarrow D(0)=0 .
$$

Thus, $u \equiv 0$ is a solution, and since

$$
E^{\prime}(0)=-2, F^{\prime}(0)=0 \Rightarrow D^{\prime}(0)=\frac{\mu^{2}(1+\varkappa)-\lambda^{2}}{\lambda},
$$


it follows that the solution $u \equiv 0$ is unstable for $\mu^{2}(1+\varkappa)>\lambda^{2}$ and asymptotically stable for $\mu^{2}(1+\varkappa)<\lambda^{2}$.

Let $z \neq 0, u \neq 0$. We will seek a solution to Eq. (4.8) of the form $z=$ const:

$$
\begin{gathered}
B(z) z^{2}+\left(1+\lambda^{2}-\mu^{2}\right) A(z) z-\mu^{2}(B(z)+2 \varkappa W(z))=0 \Leftrightarrow \mu^{2}=f(z), \\
f(z)=\frac{B(z) z^{2}+\left(1+\lambda^{2}\right) A(z) z}{A(z) z+B(z)+2 \varkappa W(z)} .
\end{gathered}
$$

Let us find $\lim _{z \rightarrow \infty} f(z)$. To start with, we estimate the asymptotics of $w_{A, B}(z)$ :

$$
w_{A}(z)=1+z+\frac{\lambda^{2}}{2 z^{2}}+O\left(\frac{1}{z^{3}}\right), w_{B}(z)=-1+z+\frac{\lambda^{2}}{2 z^{2}}+O\left(\frac{1}{z^{3}}\right), z \rightarrow \infty .
$$

Then

$$
f(z)=\frac{\lambda^{2}+O\left(\frac{1}{z^{2}}\right)}{1+\varkappa+O\left(\frac{1}{z^{2}}\right)} \Rightarrow \lim _{z \rightarrow \infty} f(z)=\frac{\lambda^{2}}{1+\varkappa} .
$$

The graph of the function $f(z)$ has the form shown in Fig. 4.

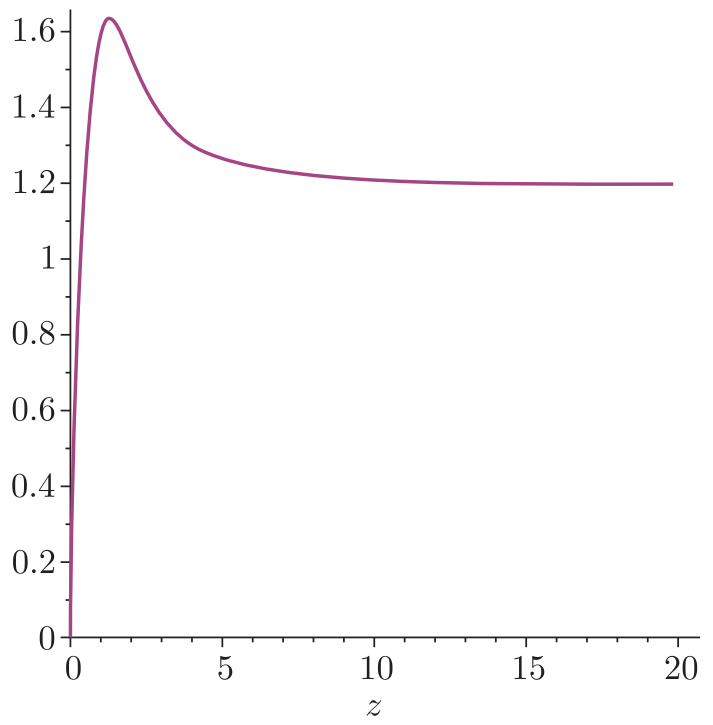

Fig. 4. The graph $\mu^{2}=f(z)$ for $z>0$.

Thus, at a sufficiently large value of the coefficient $k_{C}$ and $\forall \mu>0$, in a neighborhood of the equilibrium point there is a solution $z \equiv 0$ that is created by static friction forces at point $C$, giving rise to sets of "stagnation" from Statement 1 . For $\mu^{2} \in\left(0 ; \frac{\lambda^{2}}{1+\varkappa}\right)$ there are also solutions $u \equiv 0, z \equiv z_{1}$, with $u \equiv 0$ being asymptotically stable and $z \equiv z_{1}$ unstable. For $\mu^{2} \in\left(\frac{\lambda^{2}}{1+\varkappa} ; \mu_{\max }^{2}\right)$ there are solutions $u \equiv 0, z \equiv z_{1}, z \equiv z_{2}, z_{2}>z_{1}$, with $u \equiv 0, z \equiv z_{1}$ being unstable and $z \equiv z_{1}$ asymptotically stable.

Examples of phase portraits are given in Fig. 5.

\section{References}

[1] Chaplygin, S. A., On the Theory of Motion of Nonholonomic Systems. The Reducing-Multiplier Theorem, Regul. Chaotic Dyn., 2008, vol.13, no.4, pp.369-376; see also: Mat. Sb., 1912, vol. 28, no.2, pp. 303-314. 


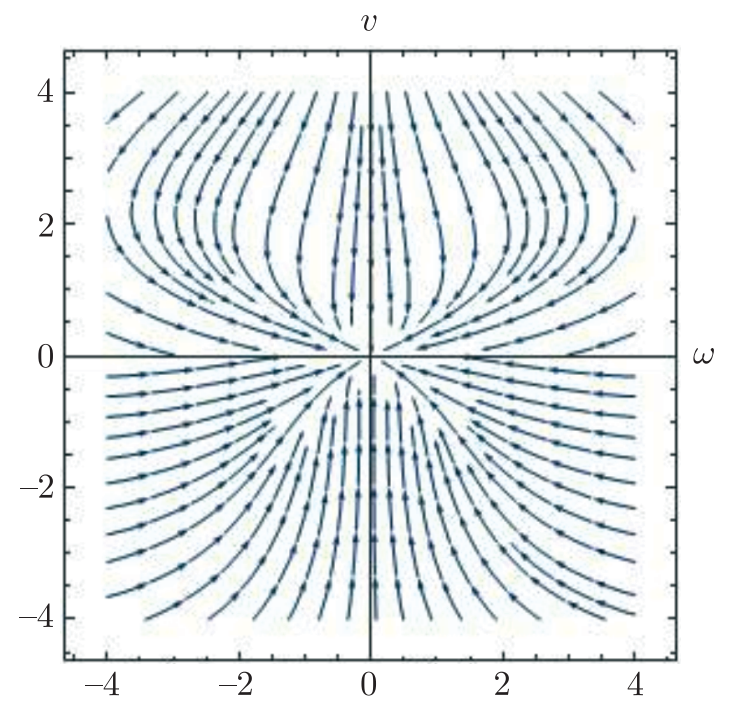

(a) the initial system

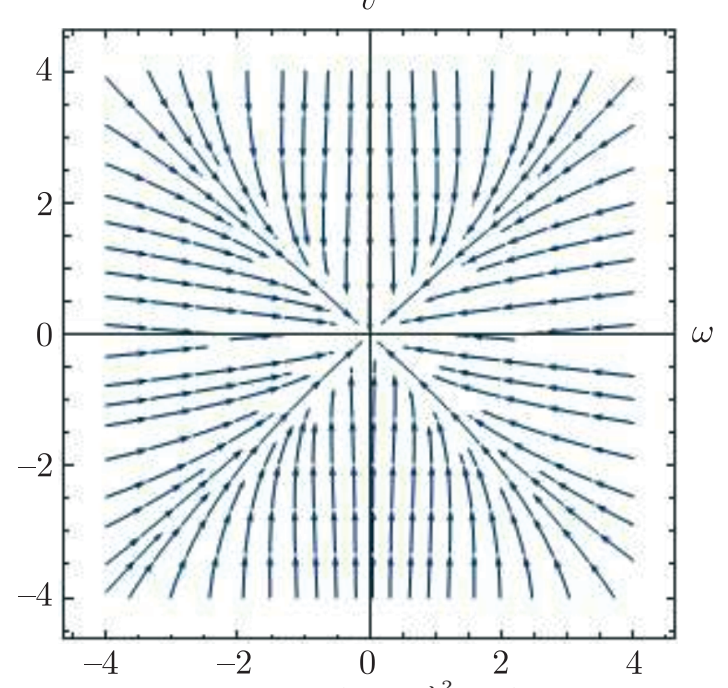

(c) $\varepsilon=0, \mu^{2} \in\left(\frac{\lambda^{2}}{1+\varkappa} ; \mu_{\max }^{2}\right)$

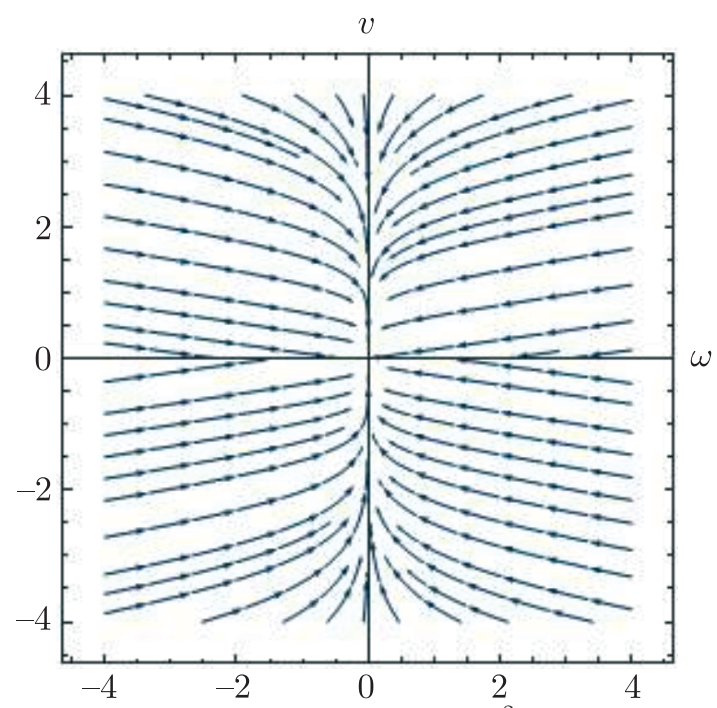

(b) $\varepsilon=0, \mu^{2} \in\left(0 ; \frac{\lambda^{2}}{1+\varkappa}\right)$

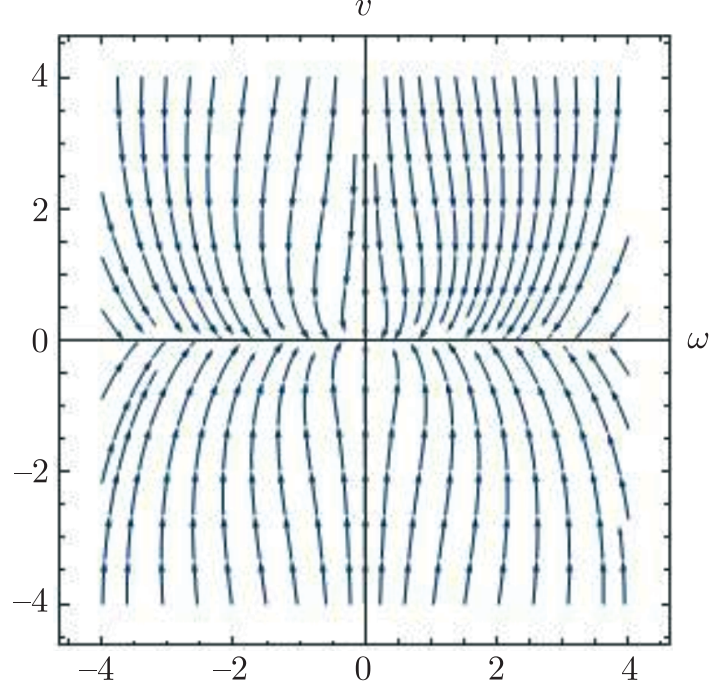

(d) $\varepsilon=0, \mu>\mu_{\max }$

Fig. 5. Phase portraits for $h=0$.

[2] Neimark, Ju. I. and Fufaev, N. A., Dynamics of Nonholonomic Systems, Trans. Math. Monogr., vol. 33, Providence, R.I.: AMS, 1972.

[3] Levi-Civita, T., Sulla stabilità delle lavagna a cavalletto, Periodico de Mathematiche (4), 1924, vol.4, pp. 59-73.

[4] Karapetyan, A. V. and Shamin, A. Yu. On the Movement of the Chaplygin Sleigh on a Horizontal Plane with Dry Friction, J. Appl. Math. Mech., 2019, vol. 83, no. 2, pp. 251-256; see also: Prikl. Mat. Mekh., 2019, vol. 83, no. 2, pp. 228-233.

[5] Ivanov, A. P., Fundamentals of the Theory of Systems with Friction, Moscow-Izhevsk: R\&C Dynamics, Institute of Computer Science, 2011 (Russian).

[6] Sumbatov, A.S. and Yunin, E.K., Selected Problems of Mechanics of Systems with Dry Friction, Moscow: Fizmatlit, 2013 (Russian).

[7] Kuleshov, A. S., Treschev, D. V., Ivanova, T. B., and Naimushina, O. S., A Rigid Cylinder on a Viscoelastic Plane, Nelin. Dinam., 2011, vol. 7, no. 3, pp. 601-625 (Russian). 